Relations industrielles

Industrial Relations

\title{
Un Canada bilingue et biculturel
}

\section{Université de Montréal}

Volume 23, numéro 3, 1968

URI : https://id.erudit.org/iderudit/027927ar

DOI : https://doi.org/10.7202/027927ar

Aller au sommaire du numéro

Éditeur(s)

Département des relations industrielles de l'Université Laval

ISSN

0034-379X (imprimé)

1703-8138 (numérique)

Découvrir la revue

Citer cet article

Université de Montréal (1968). Un Canada bilingue et biculturel. Relations industrielles / Industrial Relations, 23(3), 484-486.

https://doi.org/10.7202/027927ar
Résumé de l'article

En 1965, l’Université de Montréal présentait un mémoire à la Commission d'enquête sur le bilinguisme et le biculturalisme. Nous avons crû bon en reproduire quelques extraits.
Tous droits réservés (C) Département des relations industrielles de l'Université Laval, 1968
Ce document est protégé par la loi sur le droit d'auteur. L'utilisation des services d'Érudit (y compris la reproduction) est assujettie à sa politique d'utilisation que vous pouvez consulter en ligne.

https://apropos.erudit.org/fr/usagers/politique-dutilisation/ 


\title{
Un Canada bilingue et biculturel
}

\section{Université de Montréal}

\author{
En 1965, PUniversité de Montréal présentait un mé- \\ moire à la Commission d'enquête sur le bilinguisme et le \\ biculturalisme. Nous avons crâ bon en reproduire quelques \\ extraits.
}

Le Canada français étant ce qu'il est et affirmant sa détermination d'évoluer comme tel, le bilinguisme et le biculturalisme sont les éléments essentiels de l'existence d'un Canada uni. Le bilinguisme et le biculturalisme sont des objectifs réalisables si tous ceux qui le veulent se mettent résolument à la tâche et prennent les moyens de parvenir à cette fin. Selon les lieux et les circonstances, il faudra stimuler la bonne volonté des uns, vaincre les résistances et les préjugés des autres, mettre à la disposition de tous des maîtres compétents et des méthodes éprouvées et, dans tous les cas, fonder ses efforts sur l'intérèt qui est le mobile de tous les actes humains. L'effort sera long et soutenu si on veut travailler en profondeur et que les résultats soient durables.

Dans l'état actuel des choses, des réformes s'imposent dans les institutions car leur structure présente en empêche plusieurs de jouer le rôle qui leur revient dans un pays bilingue et biculturel. S'il est des esprits préparés à comprendre l'utilité et l'urgence de procéder aux réformes nécessaires, il en est d'autres, et sans doute plus nombreux, qui s'y refusent ou pour qui elles sont indifférentes. De là la nécessité, d'une part, de profiter de la bonne volonté des uns afin de procéder immédiatement aux tâches les plus urgentes et, d'autre part, de projeter à plus ou moins longue échéance la réalisation des autres qui ne deviendront possible que lorsque la réforme se sera faite dans les esprits.

Il appartient d'abord au gouvernement fédéral et aux institutions qui en dépendent d'accélérer et d'intensifier les initiatives qu'ils ont prises en vue de généraliser le bilinguisme dans la fonction publique et dans les moyens de faire connaître et apprécier le caractère biculturel du Canada.

Parmi les réformes qui s'imposent, il y a d'abord celle qui assurerait des avantages aux fonctionnaires bilingues. Ils ont en effet, en plus des qualités requises pour remplir les fonctions qui leur sont confiées, celle de posséder la connaissance d'une langue additionnelle dont l'usage, s'il n'est pas toujours obligatoire, est de nature à rendre leur travail plus efficace. A la longue, on pourra ainsi parvenir à ce que le français, comme l'anglais, soit une des langues de travail des fonctionnaires postés à Ottawa et dans les milieux de langue française. On évitera de la sorte la lourdeur et la lenteur des travaux de traduction qui sont présentement de règle dans l'administration et dans les services...

A moins qu'il se destine à devenir un spécialiste, l'étudiant qui a appris un peu de français au high school n'en continue pas l'étude lorsqu'il entreprend des 
études universitaires. C'est ainsi qu'au Canada, les gens les mieux instruits ne connaissent pas l'une des deux langues officielles de leur pays. Pourquoi s'étonner alors que, sauf de rares exceptions, les échelons supérieurs de la fonction publique et de l'administration dans l'entreprise privée ne soient occupés que par des unilingues? Quand on songe aussi au nombre de professeurs d'université qui sont dans la même situation, il nous vient à l'esprit la pensée que l'élite intellectuelle du pays est étrangère à ce qui se passe dans une partie importante du Canada; elle n'en connait pas plus que ne peuvent en savoir les gens moins instruits en lisant les journaux de langue anglaise. Un dialogue intelligent entre le Canada français et le reste du pays n'est guère possible dans ces conditions.

Puisque l'intérêt mène le monde, il convient de faire comprendre que c'est une qualité que d'être bilingue. Quiconque l'a acquise mérite que dans un concours, on le préfère à celui qui ne la possède pas. Si la fonction publique fédérale adoptait cette règle et l'appliquait jusque dans les échelons supérieurs de l'administration, elle inciterait dès maintenant les étudiants à acquérir cette qualité qui les distinguerait parmi les concurrents à des postes dont on décréterait que les titulaires doivent être bilingues. L'entreprise privée ferait bien aussi de suivre la mème voie dans le cas de ses employés qui viendront s'établir dans le Québec. Toutes ces mesures auront pour résultat de rendre attrayante l'étude du français même dans les provinces qui ne la rendraient pas obligatoire.

L'idéal serait que, dans un pays bilingue, l'étude des deux langues officielles soit obligatoire. Une telle mesure se heurte à des obstacles dont quelques-uns paraissent, à l'heure actuelle, insurmontables. Le premier de ces obstacles, et le plus important, est celui de l'inutilité, pour un grand nombre, de cette étude. On ne le vaincra pas facilement si jamais on y parvient. Même la perspective, assez lointaine quand on l'enseigne à des enfants, de l'utilité éventuelle de la seconde langue ne suffit pas à justifier l'imposition d'une telle mesure, bien que le Québec maintienne sa politique du bilinguisme scolaire.

La minorité anglophone du Québec, de l'aveu même de plusieurs dont la bonne foi est évidente et qui appuient leurs propos sur une expérience vécue, admet avec franchise qu'elle a profité de la situation favorable que le Québec français lui a faite et qu'en retour elle n'a pas fait assez pour comprendre, en apprenant leur langue, ce que peuvent et veulent leurs compatriotes francophones. L'image qu'ils s'en faisaient, et que bien peu de choses dans le comportement des Canadiens de langue française leur permettait de croire qu'elle n'était pas fidèle, les portait à compter comme négligeable l'apport de ce groupe, minoritaire en Amérique du Nord et replié sur lui-même, au progrès matériel de la province et du pays. C'est ainsi qu'un vide s'est créé entre la direction anglaise des grandes entreprises et un prolétariat de langue française. Quiconque voulait s'élever dans l'administration devait parler l'anglais et calquer son comportement sur celui de la direction s'il ambitionnait de s'élever davantage dans la hiérarchie unilingue. Soucieux de réussir, tandis que leur système d'éducation préparait les jeunes Canadiens français aux professions libérales ou aux emplois subalternes, les Québécois d'origine étrangère suivaient plus volontiers l'exemple de leurs concitoyens de langue anglaise et grossissaient le nombre des anglophones.

Le Canada français a pris conscience de la part qu'il doit assumer et qui lui revient dans la vie économique du Québec. Parce qu'il arrive dans un monde où un bon nombre des jeux sont déjà faits et où les positions stratégiques sont déjà 
en grande partie occupées, il lui faut, de son côté, revendiquer le clroit d'être traité comme un égal et il est nécessaire, du côté de l'entreprise, de reconnaître cette situation et de faire en sorte qu'elle se liquide équitablement. L'attitude qu'elle adoptera engage l'avenir et dépasse les frontières du Québec. Le temps n'est plus où la jeunesse du Québec ne se préparait pas aux multiples tâches de l'industrie, du commerce et de l'administration. Un regard sur les statistiques de l'inscription aux études supérieures dans nos universités fait voir la courbe constamment ascendante et de plus en plus accentuée des étudiants qui se destinent à la pratique du génie, des sciences, du commerce et de l'économique. Le pourcentage annuel d'augmentation du nombre des étudiants dans les universités du Québec est supérieur à celui des autres universités canadiennes. Ce nouvel afflux de professionnels ne répond pas encore aux seuls besoins du Québec mais on peut prévoir qu'un jour l'offre sera égale à la demande. Comme ils sont et qu'ils seront encore à l'avenir francophones, l'entreprise devra s'adapter à cette situation car, même s'ils sont bilingues, l'atmosphère des usines et des bureaux ne pourra plus ètre celle qui existe aujourd'hui.

Même si l'on doit admettre qu'en Amérique, l'anglais sera toujours la langue dominante, le français ne pourra plus être entièrement laissé de côté. Si la mobilité du personnel exige qu'il soit mis en poste ailleurs au Canada, la question se pose de savoir de quelle façon les enfants venus du Québec pourront être instruits en français dans les endroits où leurs parents seront appelés à vivrc pendant un certain temps. Il s'agit aussi de savoir comment les employés appelés à occuper des fonctions dans le Québec s'adapteront aux conditions qu'imposera le bilinguisme de leur nouvel environnement. Qu'ils soient déjà bilingues ou au moins aptes à le devenir simplifiera grandement les choses.

Que ce soit donc au niveau des gouvernements, tant le fédéral que ceux des provinces, dans l'enseignement à tous ses degrés, dans l'entreprise privée, la solution qui s'impose dès maintenant exige que, de leur côté, les Canadiens de langue anglaise fassent droit aux légitimes demandes de leurs compatriotes francophones quant au bilinguisme et au biculturalisme et que ceux-ci, à leur tour, forts de la légitimité de leurs revendications et de l'appui d'un nombre grandissant de leurs compatriotes anglais qui les comprennent, ne se cantonnent pas dans un isolement dans lequel, en dépit de l'élan qui entraîne les plus dynamiques, certains semblent vouloir continuer de vivre. Le Canada tout entier est leur pays et ils doivent obtenir, même si l'échéance en paraît lointaine, que les conditions soient telles qu'ils puissent y jouir des mêmes avantages que ceux qui existent dans le Québec et que partagent leurs compatriotes de langue anglaise.

\section{RECOMMANDATIONS}

A l'intention de l'entreprise privée :

a) qu'elle admette, au Canada français, le français comme langue de travail dans ses établissements ;

b) qu'elle favorise l'usage du français dans ses relations avec la clientèle et les services gouvernementaux dans le Québec;

c) que, tout en continuant d'assurer la mobilité de son personnel dans tout le pays, elle tienne compte des conditions qui existent au Canada quant à ses employés de langue française, et au Canada français pour ses employés qui ne sont pas francophones. 